\title{
Pyogenic spondylitis
}

\author{
W. Y. Cheung • Keith D. K. Luk
}

Received: 5 September 2011 / Accepted: 29 September 2011 / Published online: 28 October 2011

(C) The Author(s) 2011. This article is published with open access at Springerlink.com

\begin{abstract}
Pyogenic spondylitis is a neurological and life threatening condition. It encompasses a broad range of clinical entities, including pyogenic spondylodiscitis, septic discitis, vertebral osteomyelitis, and epidural abscess. The incidence though low appears to be on the rise. The diagnosis is based on clinical, radiological, blood and tissue cultures and histopathological findings. Most of the cases can be treated non-operatively. Surgical treatment is required in $10-20 \%$ of patients. Anterior decompression, debridement and fusion are generally recommended and instrumentation is acceptable after good surgical debridement with postoperative antibiotic cover.
\end{abstract}

\section{Introduction and epidemiology}

Pyogenic spinal infection (pyogenic spondylitis) has afflicted humanity for thousands of years, and was found in Egyptian mummies as far back as 7000 BC [1]. It encompasses a broad range of clinical entities, including pyogenic spondylodiscitis, septic discitis, vertebral osteomyelitis, and epidural abscess. The spine is a common site of infection and accounts for $2-7 \%$ of all cases of musculoskeletal infections [2-4]. The incidence has been reported to be between 0.2 and 2 cases per 100,000 per annum and there is evidence suggesting that the incidence is rising, possibly related to the improved life expectancy of patients with chronic debilitating diseases [5-7]. Approximately $95 \%$ of pyogenic spinal infections involve the

W. Y. Cheung $\cdot$ K. D. K. Luk $(\bowtie)$

The Department of Orthopedics \& Traumatology,

The University of Hong Kong,

Pokfulam, Hong Kong SAR, China

e-mail: hrmoldk@hku.hk vertebral body and/or the intervertebral disc, with only $5 \%$ involving the posterior elements of the spine. It is primarily a disease of adults affecting predominantly people in their fifth decade of life and the age-adjusted incidence increases with every decade thereafter. Males appear to be affected twice as often as females though the reason for this is unknown [8]. Predisposing factors include diabetes mellitus, malnutrition, substance abuse, HIV infection, malignancy, long-term steroid use, chronic renal failure, liver cirrhosis and septicaemia [9-13]. Protein malnutrition results in decreased number of $\mathrm{T}$ cells with impaired cytokines productions; HIV/AIDS leads to defective neutrophils, decreased cell count and leucocyte dysfunction. Steroid mediated immunosuppression disarms both humoral and cellular response to infectious agents. All these make the individuals vulnerable to spinal infections.

\section{Pathophysiology}

Pyogenic spondylitis commonly arises from haematogenous spread of bacteria. The arterial route of spread is more common than the venous route, usually from skin, respiratory tract, genitourinary tract, gastrointestinal tract or the oral cavity, giving rise to bacteraemia. The cellular bone marrow and the sluggish but voluminous blood supply to the spine make it particularly vulnerable to bacterial inoculation and infection. The usual involvement of adjacent segments of contiguous vertebrae can be explained by the arterial supply of the axial skeleton. The same segmental artery supplies an intervening disc as well as the lower portion of the upper vertebra and the upper portion of the adjacent lower vertebra. Thus, pyogenic spondylitis typically involves two adjacent vertebrae and the intervening disc [14]. The pathophysiologies of spinal 
infection in children and adults are somewhat different. In children, the bacterial inoculation is through the persisting vascular channels in the disc space and pyogenic discitis may occur after bacteraemia. In adults, the disc is avascular, the bacteria invade the end-arterial arcades in the subchondral region adjacent to the intervertebral disc. The infection then spreads by direct extension through the end-plates to the intervertebral disc. Bacterial inoculation through the venous system may sometimes occur. In the lumbar spine, the Batson's paravertebral venous plexus may act as a potential route of infection, particularly in cases of sepsis originating from the pelvic organs [15]. In the cervical spine, the pre-vertebral pharyngeal venous plexus may act as a route for the spread of bacteria in cases of infections around the head and neck regions [16]. Bacteria may also reach the spine by direct invasion or inoculation. They include direct extension from an infected contagious source and iatrogenic inoculation. The former may occur especially in the upper cervical spine which is close to the oral pharynx. In patients suffering from nasopharyngeal carcinoma the treatment with radiotherapy rendered their pharyngeal wall thin and sometimes defective (Fig. 1), leading to infection by bacterial flora by direct invasion. Iatrogenic inoculation may occur after an invasive diagnostic or therapeutic procedure, for example, lumbar discography, and accounts for $14-26 \%$ of spinal infections [17].

Tuberculosis used to be the most common cause of spinal infections. A study at the authors' institution from 1955 to 1960 on 2,000 cases of spinal infection showed that spinal tuberculosis accounted for $59 \%$ of cases [18]. The bacteriology of spinal infection however has changed over
A

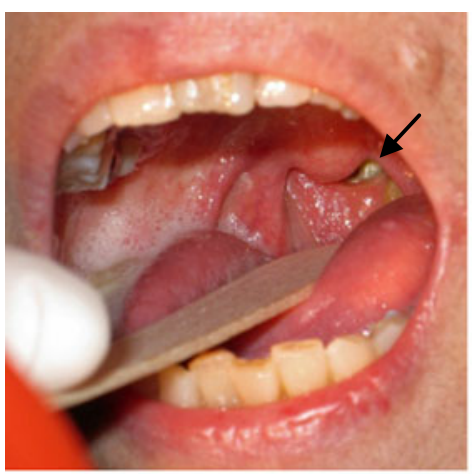

B

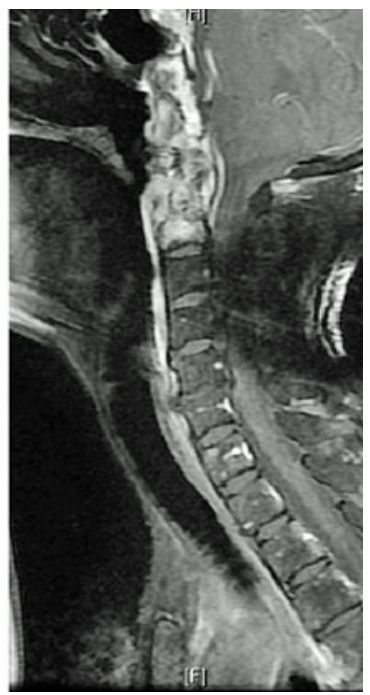

Fig. 1 a Naso-pharyngeal mucosal defect after radiotherapy for nasopharyngeal carcinoma. b Contrast MRI showed upper cervical spine infection recent decades. A similar study was repeated from 2004 to 2008 at the same institution, it was found that tuberculosis infection only accounted for $24.2 \%$ while the majority was pyogenic infections [19]. The common organisms that cause pyogenic spondylitis are Staphylococcus aureus and streptococcus species which account for more than $50 \%$ of cases $[17,19]$. Escherichia coli and proteus may be present in patients with urinary tract infections. In intravenous drug abusers, Gram negative bacilli are frequently isolated. Organisms of low virulence such as coagulase negative staphylococcus and streptococcus viridians may also cause the infections especially in immune-compromised patients. Anaerobic organisms can be inoculated directly into patients with penetrating spine trauma and are common in patients with diabetes mellitus. Salmonella osteomyelitis may be the culprit particularly in children with sickle cell anaemia. Nevertheless, in one-third of cases the infective organisms are never identified [17, 20].

The level of spinal involvement varies and infections have been recorded at all levels of the spine [21]. The most common site of infection is the lumbar spine (45-50\%), followed by the thoracic (35\%), cervical (3-20\%), and sacral regions [22]. Untreated vertebral column infections may progress to abscess formation with spread to adjacent structures, including spread into the spinal canal, and form epidural abscess. In addition, untreated infections may cause destruction of the intervertebral disc and vertebral bodies, resulting in spinal instability, vertebral body collapse with kyphosis, and neural compression. Paralysis can result from direct neural compression by bony elements or an adjacent abscess mass. It may be also caused by ischaemic damage to the spinal cord as a result of septic thrombosis or inflammatory infiltration.

\section{Clinical presentation}

The diagnosis of pyogenic spinal infection is based on clinical, radiological, and microbiological grounds. A delay in diagnosis is not uncommon and can range from two to 12 weeks, and on occasion after three months [23]. This may lead to bony destruction, kyphosis and subsequent neurological complications. The onset of symptoms is usually insidious, with back or neck pain being the most common presenting complaint, with prevalence of more than $90 \%$ of cases. Fever is typically not present and accounts for less than $20 \%$ of patients [24]. Other symptoms include nausea, vomiting, anorexia, weight loss, lethargy, and confusion. Difficulty in swallowing is also another symptom, which may be caused by cervical pyogenic spondylitis with retropharyngeal abscess. Limb weakness, numbness and sphincter dysfunction can be caused by spinal cord or cauda equina compression. Less commonly, neurological compli- 
cations can also be caused by direct neural infiltration and ischaemic damage to the spinal cord. Butler et al. reported that only $29 \%$ of patients suffering from infective spondylitis presented with evidence of neurological involvement and the majority $(79 \%)$ of them had incomplete neurological deficit with mild extremity weakness [24].

\section{Laboratory tests}

Erythrocyte sedimentation rate (ESR) is a sensitive laboratory indicator of pyogenic infection, which is positive in more than $90 \%$ of patients with spinal infections. The average ESR in patients with pyogenic spondylitis ranges from $43-87 \mathrm{~mm}$ per hour $[25,26]$. Elevation in ESR correlates with the presence of inflammatory response but it is not specific for infection. It normalises in an irregular and slow fashion even after successful treatment of an infection. Despite the nonspecific nature of an elevation in the ESR, this test provides additional data regarding the possible presence of infection and some information on response to treatment.

C-reactive protein (CRP) is an acute phase protein synthesised by hepatocytes. Only trace amounts are found in the serum of healthy patients but it increases within six hours of the onset of a bacterial infection. C-reactive protein is elevated in $90 \%$ or more of patients with spinal infection and is more specific than ESR. Although CRP and ESR are elevated after spinal surgery or infections, CRP normalises postoperatively or after appropriate treatment of an infectious process faster than the ESR. Rosahl et al. [27] showed that ESR remained markedly elevated ten days after anterior cervical discectomy and fusion with a peak on postoperative day three whereas the CRP returned to less than $50 \%$ of its peak level by postoperative day five. Therefore an elevation in CRP and/or ESR should not be taken as pathognomonic for an infection; however, these both serve as good screening and surveillance tests in the diagnosis and treatment of spinal infections.

The white blood cell (WBC) count may not be elevated in patients with a spinal infection. The WBC elevation is usually moderate, ranging from 4 to $23 \times 10^{9}$ per litre, with an average of $11.8 \times 10^{9}$ per litre. The WBC is not particularly useful in making a diagnosis of spinal infection, but should be part of an infection/fever workup as it may provide some general guidance concerning a response to treatment [28].

Given the variability in the source(s) of infection, blood cultures, urinalysis, and urine for culture should be obtained in patients suspected of having a spinal infection. About $25-59 \%$ of positive blood cultures identify the causative microorganism. A chest radiograph and sputum culture should be obtained to look for subclinical chest infection.
Antibiotic therapy should not be started until appropriate cultures have been performed unless the patient is septic or critically ill.

\section{Imaging studies}

Plain radiographs should be taken for all patients suspected of having spinal infections. These will reveal the degree of bony destruction and evaluate any corresponding coronal or sagittal mal-alignment resulting from the disease process. Early changes on plain radiographs can be inconspicuous and may develop slowly. The earliest signs are blurring of the end-plates and decrease in disc space, which happen two to eight weeks after the onset of the infection. Because many patients have degenerative changes on imaging studies, additional changes caused by infection are not so apparent in the early stage of the infectious disease. Therefore, a high index of suspicion must be maintained. After eight to 12 weeks, obvious bony destruction will be observed. If there is significant bony destruction, scalloping and collapse of the vertebral body will occur, often producing localised kyphosis (Fig. 2). Soft tissue extension with paraspinal abscess should be suspected in the presence of an abnormal soft tissue shadow, though it is less common compared with tuberculosis infections.

Radionuclide studies are more sensitive than radiographs in detecting early diseases. Three-phase technetium-99m bone scans are sensitive (sensitivity $90 \%$ ) but nonspecific (specificity 78\%) for spinal infections, particularly in older patients with some degree of spondylosis and
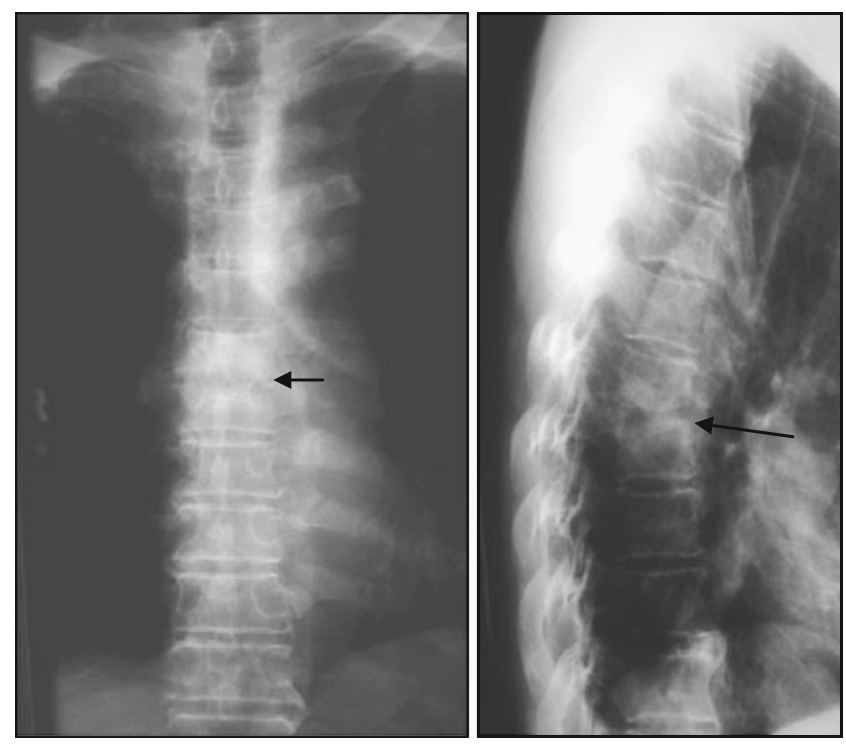

Fig. 2 T6/7 pyogenic spondylodiscitis with inter-vertebral disc and end-plates destruction and kyphosis 
degenerative disc disease [29]. Bone scans provide little anatomical detail and can also be positive in osteoporotic fractures and neoplasms. Increased activity can also be present even after the spondylitis is healed and laboratory findings return to normal. Gallium-67 citrate scans have similar sensitivity (89\%), specificity (85\%), and accuracy $(86 \%)$ as technetium scans in evaluating pyogenic spinal infections [28]. Indium 111-labeled leukocyte scintigraphy has high specificity but very low sensitivity $(17 \%)$. This is because, by the time patients are examined, many infections have progressed beyond the acute stage into a more chronic stage with a relative scarcity of leukocytes [30]. Owing to the high rate of false-negative results, it is not used routinely in diagnosing spinal infections.

Magnetic resonance imaging (MRI) is the gold standard for imaging of spinal infections. It is particularly useful in the early stages of infection when other imaging modalities are still normal (radiography) or nonspecific (nuclear medicine). Sensitivity, specificity, and accuracy of MRI are reported as $96 \%, 92 \%$, and $94 \%$, respectively [28]. The typical signal pattern of acute spinal infection on MRI is an increase in fluid signal because of marrow oedema with signal decrease in $\mathrm{T} 1$-weighted sequences and signal increase in T2-weighted sequences with contrast enhancement (Fig. 3). In most cases, the infection starts in the anterolateral vertebral body near the endplate [31]. Associated oedema typically is pronounced and affects much of the vertebral body and inter-vertebral disc [31]. Spinal neoplasms may also present with similar T1weighted and T2-weighted findings on MRI. However, the presence of disc space involvement will serve as one way to distinguish infections from neoplasms. Although MRI cannot diagnose the specific causative organism, there are some features which suggest certain infections. For example, tuberculous spondylitis is characterised by meningeal involvement, subligamentous spread of infection and paravertebral and intraosseous abscesses which can be well demonstrated with contrast MRI [32]. MRI also tends to over-estimate the amount of tissue involvement by the infection as some of the signal changes are reactive. Therefore plain X-ray and CT scan are more useful to show the amount of tissue necrosis that requires surgical debridement. In postoperative patients, the interpretation of MR images may be more difficult because there will be some increased T2-weighted signal and contrast uptake at the surgery site. After uncomplicated discectomy there is usually little signal change of the remaining disc and vertebral body and no contrast uptake of disc or vertebral body. Though there can be overlap of less florid or early discitis with normal postoperative changes, if the adjacent vertebral body shows low signal on T1-weighted images and contrast enhancement, infection is more likely [32].
MRI is the best and only reliable noninvasive method for assessing the contents of the spinal canal, particularly the epidural space and spinal cord. Epidural abscess, especially if associated with neurological deficit, is a surgical emergency. When pus collections have formed, they often are low in T1-weighted and high in T2-weighted signals. T1-gadolinium sequences will show a mass with peripheral enhancement (Fig. 3). Diffuse enhancement throughout the mass is more consistent with granulation tissue, but this finding may be unreliable and the mass should be treated as an epidural abscess if clinically warranted [31].

In established cases of pyogenic spondylitis, follow-up MRI to assess treatment outcome can be confusing. If MRI is repeated early in the course of successful treatment, the patient may already be improving clinically while the imaging findings continue to worsen with more extensive disease spread and bone destruction. This is common as MRI is not sensitive for the onset of reparative changes. The earliest sign of healing on MRI is reduced soft tissue inflammation and decrease in contrast enhancement. This typically occurs sometime between a few weeks and a few months after commencement of appropriate treatment. The absence of contrast enhancement and return to normal signal pattern are reliable signs of completed healing, though some degree of contrast uptake may be present for more than a year. Persistence of abnormal contrast uptake is more common in the disc compared with the vertebral body. The marrow pattern can return to normal after successful treatment. However with the reparative process being highlighted by fibrosis and sclerosis, low T1-weighted and T2-weighted signals may be seen. High T1 fatty marrow may also replace some areas of previously affected marrow.

$18 \mathrm{~F}$-fluorodeoxyglucose (18F-FDG) is known to accumulate at sites of infection, inflammation, and in autoimmune and granulomatous diseases. The utility of FDGpositron emission tomography (PET) for the diagnosis of musculoskeletal infection has been reported for orthopaedic diseases. Sixty patients with suspected chronic musculoskeletal infection involving the central skeleton or the peripheral skeleton were studied with FDG-PET, and it was found to be highly accurate as a single technique for the evaluation of chronic musculoskeletal infections [33]. FDG-PET has also been shown as a useful adjunct to imaging for the diagnosis of spondylodiscitis [34]. Schmitz et al. reported that all patients with spinal infection confirmed by histopathology had positive FDG-PET imaging [35]. Stumpe et al. reported the utility of FDG-PET for differentiation of degenerative and infectious endplate abnormalities in the lumbar spine that were detected on MRI [36]. PET did not show FDG uptake in the intervertebral spaces of any patient with degenerative disease. FDG-PET findings were true positive at all five levels with disc-space infection. The sensitivity and specificity for MRI 
Fig. 3 a T1-weighted image. b T2-weighted image. c T1 weighted with contrast image on a patient showing features of pyogenic spondylitis at $\mathrm{L} 4 / 5$ with epidural abscess
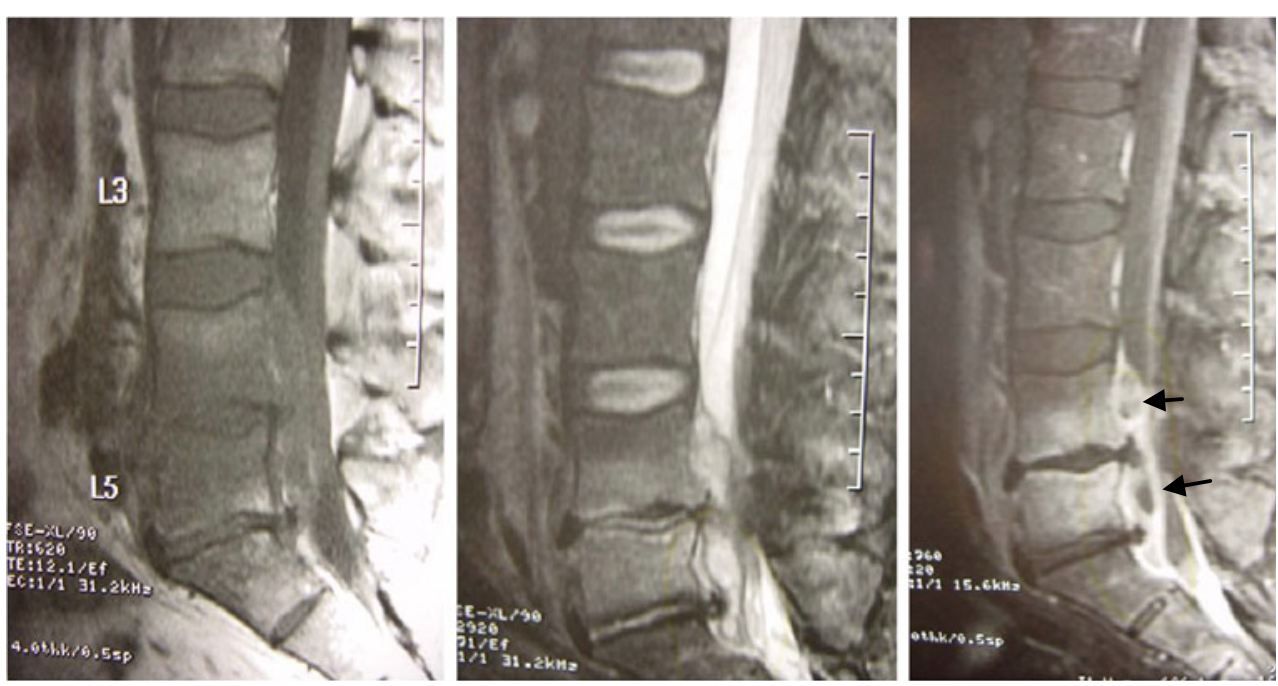

in detecting disc-space infection were $50 \%$ and $96 \%$, and were $100 \%$ and $100 \%$ for FDG-PET, respectively.

\section{Biopsy}

A clinical diagnosis of pyogenic spondylitis can be made in patients with positive blood cultures and compatible clinical history in combination with corresponding changes on laboratory and imaging studies. The definitive diagnosis of pyogenic spondylitis can only be made on microscopic or bacteriological examination and culture of the infected tissues. In the absence of a positive blood culture, the recommended algorithm of management in a stable patient is to withhold antibiotics until a fluoroscopic or CT-guided percutaneous biopsy is done. Endoscopic biopsy is another option which allows the biopsy to be done under visualisation and debridement of the infected tissues [37]. The biopsy specimens should be sent for Gram smear, aerobic and anaerobic cultures, tuberculosis culture and polymerase chain reaction, fungal culture and histopathology. The accuracy of percutaneous vertebral biopsy in patients with infective spondylitis has been reported to be about $70 \%$. A core biopsy is preferable to fine needle aspiration $[17,22]$. A negative result may be due to insufficient tissue or administration of antibiotics. If the initial biopsy is negative and withholding antibiotics is considered safe clinically, a further biopsy can be attempted. If a fluoroscopic or CTguided percutaneous biopsy fails to identify the organism, an open biopsy should be performed.

\section{Non-surgical treatments}

A majority of patients with infective spondylitis can be treated non-operatively. After septic work-up and tissue biopsy, antibiotic treatment should be started. In our centre, empirical antibiotics comprising penicillin or first generation cephalosporin are used to cover the common infective organisms, namely, staphylococcus and streptococcus. For immune-compromised patients and intravenous drug abusers, broader spectrum antibiotics, e.g. third generation cephalosporin with better gram negative bacteria coverage, is added. Clindamycin, vancomycin, quinolones, tetracycline and cotrimoxazole also have good bone penetration properties and should be considered when treating spinal infections, especially for patients sensitive to beta-lactam antibiotics. Besides good bone concentration, rifampicin is active against biofilm embedded bacteria and has synergistic effects with other beta-lactam antibiotics, making it particularly useful in patients with Gram positive coccal infections with implants [32]. The choice of antibiotic is adjusted according to the subsequent bacterial culture results. Consultation with microbiologists is important to optimise antibiotic cover and to identify possible sources of bacteraemia. The optimal duration of antibiotic therapy is not well defined, with several studies recommending six to eight weeks of intravenous therapy and others recommending only four weeks. Antibiotic therapy for less than four weeks may result in an unacceptably high recurrence rate [22]. In our centre, we treat with intravenous antibiotics until the CRP is normal which usually takes about two to four weeks, then change to oral antibiotics for a total of three months.

Bed rest is recommended in the early phase until the acute pain improves. Ambulation in an appropriate cast or brace is then recommended. External immobilisation helps to stabilise the spine, decrease pain and prevent deformity. The duration of bracing ranges from three to four months depending on the amount of bony destruction or deformity. Progression of deformity may be noted in approximately $30 \%$ of patients during the first six to eight weeks [38]. 
The effectiveness of non-operative treatment can be assessed by following the clinical response for any improvement in pain and pyrexia, laboratory investigations, and radiographic imaging. With a resolving spinal infection, laboratory studies should show declining CRP and ESR values and imaging studies should demonstrate a halt in cortical destruction with subsequent osseous fusion. If the infective parameters remain persistently elevated with intravenous antibiotics, a repeat imaging study should be considered to look for any abscess collection and longer course of intravenous therapy may be indicated [39]. Nonoperative treatment leads to a clinical success in approximately $75 \%$ of patients. At six to 24 months after the onset of symptoms, most patients successfully treated without surgery experience spontaneous interbody fusion. However, a relapse rate of $14 \%$ and other complications including worsening symptoms, neurological deficit, spread of the infection, and significant spinal deformity have been reported [40].

\section{Surgical treatments}

Only $10-20 \%$ of patients suffering from pyogenic spondylitis require open surgery. Surgical treatment is absolutely indicated in patients with spinal cord or cauda equina compression with progressive neurological deficits. Emergency surgical decompression should be considered in this situation as the prognosis for neurological recovery is better compared to non-operative management. Relative indications for surgery include uncertain diagnosis for which open surgery can obtain tissues for bacteriological and histological confirmation, failed conservative treatment with lack of clinical improvement after two to three weeks of antibiotic treatment or significant progressive spinal deformity with biomechanical instability. Because spinal infections commonly involve the vertebral body, an anterior surgical approach is usually recommended. It allows radical debridement of the infection site and placement of a structural bone graft or cage to reconstruct the vertebral column. Transoral or retropharyngeal approaches may be necessary to reach the craniocervical junction. In the subaxial cervical spine, a standard Smith-Robinson approach can provide excellent exposure. Lesions involving the thoracic spine can be approached anteriorly by thoracotomy. In the lumbar spine, a retroperitoneal approach is preferable to a transperitoneal approach because it avoids potential seeding of the peritoneal cavity. Posterolateral extra-cavitary approaches in the thoracic and lumbar spine are becoming more popular for dealing with anterior vertebral column pathologies including infection. They obviate the need for a direct anterior thoracic or lumbar approach but may be technically more difficult especially when there is extensive epidural adhesion due to the infection. Rarely, patients may present primarily with epidural abscess without bone destruction, and the epidural abscess can usually be drained with posterior approach and laminectomy.

Traditionally, the operative treatment for infective spondylitis is anterior decompression and debridement, followed by an anterior fusion. The infected tissues are thoroughly debrided and the abscesses drained. The debridement should extend back to healthy bleeding bone to allow for subsequent tissue healing. The objective of debridement is to accelerate the natural course of healing by evacuating the bulk of the infected tissue and opening vascular channels from the subchondral bone to facilitate antibiotic delivery and speed up the process of invasion by the reparative granulation tissues. After extensive debridement of the infected tissue, structural bone or cage grafting may be performed simultaneously or at a delayed time. Anterior strut grafting with autograft, allograft, and titanium cages has been performed safely and successfully in the presence of infection after most of the purulent material has been removed [41]. In cases of cervical infection with gross purulence, some surgeons have recommended anterior debridement alone with application of a halo device after surgery, followed by delayed secondary debridement and anterior fusion after a period of seven to 14 days of intravenous antibiotic treatment [42]. Anterior spinal fusion permits more rapid bony healing with less vertebral column collapse, faster patient rehabilitation, and a diminished incidence of reactivation of the infection compared to nonoperative treatment [43]. Fang et al. reported a series of 39 patients who underwent an anterior decompression and debridement with fusion for pyogenic spondylitis. Of 30 patients with an average follow-up of five years, all had symptoms improved after surgery and bone fusion was achieved in $93 \%$, with the time to fusion averaging six to seven months. There was one postoperative wound complication and one case of recurrent infection. The authors concluded that anterior debridement and fusion give good early and long-term results, with rapid recovery of the patient, low morbidity, and low mortality [44].

Bacteria possess a layer of polysaccharides external to the cell wall. When the bacterium encounters an inanimate object, it will attach to the surface, multiply and form a layer of exo-polysaccharides known as glycocalynx, which serves as cement to bind the bacteria to the implant, making them resistant to antimicrobial agents. Because of this, the use of instrumentation in the setting of spinal infection has been controversial. Recently many studies have reported the safety and efficacy of internal fixation in the setting of active spinal osteomyelitis, provided that debridement of the infected tissue is complete and an appropriate course of antibiotic therapy is followed [45-47]. Internal fixation 
maintains spinal stability and alignment, prevents postoperative kyphotic deformity and promotes bony fusion. Muckley et al. [48] reported three patients treated with radical decompression and debridement with interbody fusion and anterior spinal fixation. There was no recurrence of infection or loss of reduction during follow-up. Although anterior-only surgery can be performed, combined anteriorposterior surgery provides a much stronger spinal construction with greater spinal stability. General indications for combined anterior-posterior surgery include the presence of significant kyphotic or scoliotic deformity, a location of infection that is not amenable to bracing after anterior surgery alone, and multiple-level involvement especially if two or more vertebrae are spanned. In order to mobilise the patients more rapidly and eliminate complications associated with bed rest and prolonged hospitalisation, the use of a more aggressive single-stage strategy is recommended [49]. In patients treated with single-stage debridement, arthrodesis, and internal fixation, Przybylski and Sharan found that instrumentation displacements occur in $6.6 \%$, deep wound infection in $6.6 \%$, and superficial infection in $2.8 \%$ [50]. Safran and colleagues reported on ten patients with infective spondylitis who underwent one stage anterior decompression and debridement as well as posterior stabilisation. All patients were mobilised within the two days after surgery, with eradication of the infection [51].

Materials for anterior column reconstruction of the spine after radical debridement have predominantly included autograft, allograft, and titanium mesh cages. The advantage of autograft in anterior spinal reconstruction is the reduction of potential risk associated with introducing nonliving material into an infected tissue bed. However, it is associated with added operative time, difficulty in placement, and donor site morbidity. McGuire and Eismont found that autogenous bone graft at the time of primary debridement for pyogenic spondylitis has led to solid union in $96 \%$ of cases with eradication of infection in all patients though with mild collapse of the interbody grafts [52].

Allograft has been successfully used in patients with spinal osteomyelitis. The principal advantages of allograft are the absence of donor site morbidity, the abundant supply, and the numerous shapes and sizes that can accommodate the geometry of a given anterior column defect. Schuster et al. reported a series of 47 patients who underwent anterior surgical decompression in which fresh-frozen structural allograft was used after aggressive debridement, followed by a posterior stabilisation procedure. During the average follow-up period of 17 months, no patient required reoperation for a persistent infection or failure of instrumentation/ allograft. These investigators concluded that structural allograft in combination with aggressive tissue debridement and adjuvant antibiotic therapy is a safe and effective therapy in cases of infective spondylitis requiring surgery [53].
In addition to structural autograft and allograft, titanium mesh cages filled with autogenous bone have been used for anterior column reconstruction. Several authors have concluded that the use of metallic implants after radical debridement of the infected spine does not lead to persistence or recurrence of the infection [54, 55]. Fayazi et al. reported on a series of 11 patients who underwent spinal reconstruction using cylindrical titanium mesh cages. There was no evidence of recurrence or residual infection in any of their patients. There was one case of pseudoarthrosis and one instrumentation failure [56]. Liljenqvist et al. reported on 20 patients who were surgically treated with anterior column reconstruction using an expandable titanium cage. All cages fused, and all infections were eradicated. There was no evidence of cage dislocation, migration, or subsidence [54]. Hee et al. reported five patients who had spinal reconstruction with titanium cages and posterior instrumentation. All infections were successfully treated with adequate correction of sagittal and coronal alignment [55].

Open Access This article is distributed under the terms of the Creative Commons Attribution Noncommercial License which permits any noncommercial use, distribution, and reproduction in any medium, provided the original author(s) and source are credited.

\section{References}

1. MacDonald JW (2002) Management of spinal infection. Curr Orthop 16:462-470

2. Tyrrel PNM, Cassar-Pollucino VN, McCall IW (1999) Spinal infection. Eur Radiol 9:1066-1077

3. Stabler A, Reiser MF (2001) Imaging of spinal infection. Radiol Clin North Am 39:115-135

4. Danner RL, Hartmann BJ (1987) Update of spinal epidural abscess: 35 cases and review of the literature. Rev Infect Dis 9:265-274

5. Sapico FL, Montgomerie JZ (1979) Pyogenic vertebral osteomyelitis: report of nine cases and review of the literature. Rev Infect Dis 1:754-776

6. Kapeller P, Fazekas F, Krametter D et al (1997) Pyogenic infectious spondylitis: clinical, laboratory and MRI features. Eur Neurol 38:94-98

7. Hopkinson N, Stevenson J, Benjamin S (2001) A case ascertainment study of septic discitis: clinical, microbiological and radiological features. QJM 94:465-470

8. Tali ET (2004) Spinal infections. Eur J Radiol 50:120-133

9. Del Curling O, Jr GDJ, McWhorter JM (1990) Changing concepts in spinal epidural abscess: a report of 29 cases. Neurosurg 27:185-192

10. Sampath P, Rigamonti D (1999) Spinal epidural abscess: a review of epidemiology, diagnosis and treatment. J Sp Disord 12:89-93

11. Reihsaus E, Waldbaur H, Seeling W (2000) Spinal epidural abscess: a meta-analysis of 915 patients. Neurosurg Rev 23:175-204

12. Boszcyk BM, Krause P, Bolay H, Hohmann F, Mayer HM (2000) Spinal epidural abscess following blunt pelvic trauma. Eur Spine J 9:80-84 
13. Soehle M, Wallengang $T$ (2002) Spinal epidural abscesses: clinical manifestations, prognostic factors and outcomes. Neurosurg 51:79-85

14. Sapico FL, Montgomerie JZ (1990) Vertebral osteomyelitis. Infect Dis Clin North Am 4(3):539-550

15. Batson OV (1976) The vertebral system of veins as a means for cancer dissemination. Prog Clin Can 3:1-18

16. Wiley AM, Trueta J (1959) The vascular anatomy of the spine and its relationship to pyogenic vertebral psteomyelitis. J Bone Joint Surg Br 41-B:796-809

17. Govender S (2005) Spinal infection. J Bone Joint Surg Br 87B: $1454-1458$

18. Leong JC, Fraser RD (1996) Spinal infection. In: Diesel SW (ed) The lumbar spine, 2nd ed. WB Saunders Co

19. Yee DKH, Samartzis D, Wong YW, Luk KDK, Cheung KMC (2010) Infective spondylitis in Southern Chinese. A descriptive and comparative study of ninety-one cases. Spine 35:635-641

20. Sapico FL (1996) Microbiology and antimicrobial therapy of spinal infections. Orthop Clin North Am 25:9-13

21. Wood GW II, Edmonson AS (1989) Osteomyelitis of the spine. Spine 3(3):461-493

22. Jaramillo-de la Torre JJ, Bohinski RJ, Kuntz C (2006) Vertebral osteomyelitis. Neurosurg Clin N Am 17(3):339-351

23. Perronne C, Saba J, Behloul Z et al (1994) Pyogenic and tuberculous spondylodiskitis (vertebral osteomyelitis) in 80 adult patients. Clin Infect Dis 19(4):746-750

24. Butler JS, Shelly MJ, Timlin M, Powderly WG, O’Byrne JM (2006) Nontuberculous pyogenic spinal infection in adults A 12-year experience from a tertiary referral center. Spine 31:2695-2700

25. Carragee EJ (1997) Pyogenic vertebral osteomyelitis. J Bone Joint Surg 79:874-880

26. Carragee EJ, Kim D, van der Vlugt T, Vittum D (1997) The clinical use of erythrocyte sedimentation rate in pyogenic vertebral osteomyelitis. Spine 22:2089-2093

27. Rosahl SK, Gharabaghi A, Zink P, Samii Ml (2000) Monitoring of blood parameters following anterior cervical fusion. J Neurosurg 92:169-174

28. An HS, Seldomridge A (2006) Spinal infections. Diagnostic tests and imaging studies. Clin Orthop Relat Res 444:27-33

29. Rodiek SO (2001) Diagnostic methods in spinal infections. Radiology 41:976-986

30. Whalen JL, Brown ML, McLeod R et al (1991) Limitations of indium leukocyte imaging for the diagnosis of spine infections. Spine 16:193-197

31. Modic MT, Feiglin DH, Piraino DW et al (1985) Vertebral osteomyelitis: assessment using MR. Radiology 157:157-166

32. Norden CW (1998) Lessons learned from animal models of osteomyelitis. Rev Infect Dis 10:103-110

33. de Winter F, van de Wiele C, Vogelaers D et al (2001) Fluorine-18 fluorodeoxyglucose-position emission tomography: a highly accurate imaging modality for the diagnosis of chronic musculoskeletal infections. J Bone Joint Surg Am 83:651-660

34. James SL, Davies AM (2006) Imaging of infectious spinal disorders in children and adults. Eur J Radiol 58:27-40

35. Schmitz A, Risse JH, Grunwald F et al (2001) Fluorine-18 fluorodeoxyglucose positron emission tomography findings in spondylodiscitis: preliminary results. Eur Spine J 10:534-539

36. Stumpe KD, Zanetti M, Weishaupt D et al (2002) FDG positron emission tomography for differentiation of degenerative and infectious endplate abnormalities in the lumbar spine detected on MR imaging. Am J Roentgenol 179:1151-1157
37. Yang SC, Fu TS, Chen LH, Niu CC, Lai PL, Chen WJ (2007) Percutaneous endoscopic discectomy and drainage for infectious spondylitis. Int Orthop 31:367-373

38. Frederickson B, Yuan H, Olans R (1978) Management and outcome of pyogenic vertebral osteomyelitis. Clin Orthop 131:160-167

39. Chelsom J, Solberg CO (1998) Vertebral osteomyelitis at a Norwegian university hospital 1987-97: clinical features, laboratory findings and outcome. Scand J Infect Dis 30(2):147-151

40. McHenry MC, Easley KA, Locker GA (2002) Vertebral osteomyelitis: long-term outcome for 253 patients from 7 Clevelandarea hospitals. Clin Infect Dis 34(10):1342-1350

41. Osenbach RK, Hitchon PW, Menezes AH (1990) Diagnosis and management of pyogenic vertebral osteomyelitis in adults. Surg Neurol 33(4):266-275

42. Acosta FL Jr, Chin CT, Quinones-Hinojosa A et al (2004) Diagnosis and management of adult pyogenic osteomyelitis of the cervical spine. Neurosurg Focus 17(6):E2

43. Kemp HB, Jackson JW, Jeremiah JD et al (1973) Anterior fusion of the spine for infective lesions in adults. J Bone Joint Surg Br 55 (4):715-734

44. Fang D, Cheung KM, Dos Remedios ID et al (1994) Pyogenic vertebral osteomyelitis: treatment by anterior spinal debridement and fusion. J Spinal Disord 7(2):173-180

45. Carragee EJ (1997) Instrumentation of the infected and unstable spine: a review of 17 cases from the thoracic and lumbar spine with pyogenic infections. J Spinal Disord 10(4):317-324

46. Lee MC, Wang MY, Fessler RG et al (2004) Instrumentation in patients with spinal infection. Neurosurg Focus 17(6):E7

47. Linhardt O, Matussek J, Refior HJ, Krödel A (2007) Long-term results of ventro-dorsal versus ventral instrumentation fusion in the treatment of spondylitis. Int Orthop 31:113-119

48. Muckley T, Schutz T, Schmidt MH et al (2004) The role of thoracoscopic spinal surgery in the management of pyogenic vertebral osteomyelitis. Spine 29(11 Suppl):E227-E233

49. Matsui H, Hirano N, Sakaguchi Y (1998) Vertebral osteomyelitis: an analysis of 38 surgically treated cases. Eur Spine J 7(1):50-54

50. Przybylski GJ, Sharan AD (2001) Single-stage autogenous bone grafting and internal fixation in the surgical management of pyogenic discitis and vertebral osteomyelitis. J Neurosurg 94(1 Suppl): $1-7$

51. Safran O, Rand N, Kaplan L et al (1998) Sequential or simultaneous, same-day anterior decompression and posterior stabilization in the management of vertebral osteomyelitis of the lumbar spine. Spine 23(17):1885-1890

52. McGuire RA, Eismont FJ (1994) The fate of autogenous bone graft in surgically treated pyogenic vertebral osteomyelitis. J Spinal Disord 7(3):206-215

53. Schuster JM, Avellino AM, Mann FA et al (2000) Use of structural allografts in spinal osteomyelitis: a review of 47 cases. J Neurosurg 93(1 Suppl):8-14

54. Liljenqvist U, Lerner T, Bullmann V et al (2003) Titanium cages in the surgical treatment of severe vertebral osteomyelitis. Eur Spine J 12(6):606-612

55. Hee HT, Majd ME, Holt RT et al (2002) Better treatment of vertebral osteomyelitis using posterior stabilization and titanium mesh cages. J Spinal Disord Tech 15(2):149-156

56. Fayazi AH, Ludwig SC, Dabbah M et al (2004) Preliminary results of staged anterior debridement and reconstruction using titanium mesh cages in the treatment of thoracolumbar vertebral osteomyelitis. Spine J 4(4):388-395 